The ring attractor theory also predicted that the activity packet across the ring attractor should be coherent and self-consistent, even in the absence of external inputs. Peyrache et al. ${ }^{1}$ evaluated head direction ensemble activity during waking and sleep states, finding coherent, but drifting, representations (what they call the virtual gaze) during sleep. This is clear evidence that functional connectivity in head direction networks is capable of continuously representing adjacent headings in terms of functionally adjacent (overlapping) network states and that the network states that represent different directions are robust across brain states. During slow wave sleep, this coherent representational drift was maintained at a much faster timescale than during waking or REM sleep, which is reminiscent of the temporal compression of hippocampal sequence replay events during slow-wave sleep ${ }^{15}$. Whether the drift in head direction representation during sleep corresponds to any other representations that replay during sleep (such as in hippocampus and neocortex) is a very intriguing, but open, question.

Both of the brain structures that Peyrache et al. ${ }^{1}$ recorded from exhibited dynamics consistent with attractor theory, but the coordinated activity in ADN exhibited stronger internal coherence than that in PoS. Furthermore, the correlations between ADN-PoS neuron pairs were stronger than the correlations between PoS-PoS neuron pairs. Other researchers have suggested that the ring attractor that underlies the head direction representation occurs upstream of $\mathrm{ADN}$ and $\mathrm{PoS}$ and is passed down to them ${ }^{3}$. The data from Peyrache et al. ${ }^{1}$ suggest that the head direction information is passed down from $\mathrm{ADN}$ to PoS, but suggest that $\mathrm{ADN}$ or its upstream inputs do contain the ring attractor dynamics to generate the internal sense of direction. Further causal manipulations will be required to determine exactly which brain structures internally generate the head direction signal.

The importance of the findings presented by Peyrache et al. ${ }^{1}$ is that their observation of coherent representations during both waking and sleep states implies an internally generated head direction representation. Their data support the well-developed theory of attractor-based internal generation of the orientation sense in the head direction system. The attractor network framework provides a clear context in which many already established findings regarding the head direction system have been understood. Sometimes, theory suggests incisive experiments that yield results that force us, as researchers, to re-orient the trajectory of our inquiries.
In this case, the elegant findings presented by Peyrache et al. ${ }^{1}$ embody a striking confirmation that these theories have headed us in the right direction.

\section{COMPETING FINANCIAL INTERESTS}

The authors declare no competing financial interests.

1. Peyrache, A., Lacroix, M.M., Perersen, P. \& Buzsáki, G. Nat. Neurosci. 18, 569-575 (2015).

2. Taube, J.S., Muller, R.U. \& Ranck, J.B. Jr. J. Neurosci. 10, 420-435 (1990).

3. Taube, J.S., Goodridge, J.P., Golob, E.J., Dudchenko, P.A. \& Stackman, R.W. Brain Res. Bull. 40, 477-484 (1996).

4. Sharp, P.E., Blair, H.T. \& Cho, J. Trends Neurosci. 24 289-294 (2001).

5. Redish, A.D. Beyond the Cognitive Map (MIT Press, 1999).

6. Skaggs, W.E., Knierim, J.J., Kudrimoti, H.S. \& McNaughton, B.L. in Advances in Neural Information Processing Systems 173-180 (MIT Press, 1995).

7. Redish, A.D., Elga, A.N., \& Touretzky, D.S. Network: Computation in Neural Systems. 7, 671-685, (1996).

8. Zhang, K. J. Neurosci. 16, 2112-2126 (1996).

9. Knierim, J.J., Kudrimoti, H.S. \& McNaughton, B.L. J. Neurosci. 15, 1648-1659 (1995).

10. Goodridge, J.P. \& Taube, J.S. Behav. Neurosci. 109 49-61 (1995).

11. Taube, J.S. \& Burton, H.L. J. Neurophysiol. 74 1953-1971 (1995).

12. Blair, H.T., Lipscomb, B.W. \& Sharp, P.E. J. Neurophysiol. 78, 145-159 (1997).

13. Wilson, H.R. \& Cowan, J.D. Kybernetik 13, 55-80 (1973).

14. Amari, S. Biol. Cybern. 27, 77-87 (1977).

15. Nádasdy, Z., Hirase, H., Czurko, A., Csicsvari, J. \& Buzsaki, G. J. Neurosci. 19, 9497-9507 (1999).

\title{
Forming artificial memories during sleep
}

Classical conditioning, such as the association between a tone and an odor, can be induced in sleeping humans. On page 493, de Lavilléon et al. report an attempt to create artificial memories in sleeping mice. The authors took advantage of the properties of hippocampal place cells, which are thought to encode specific locations in an animal's environment. Patterns of place cell activity generated during waking are replayed during subsequent sleep. This replay occurs during sharp wave ripples, which are an oscillatory pattern of activity thought to support memory consolidation. Pairing rewarding stimulation with replay of place cell activity, the authors showed that they could indeed induce artificial memories in sleeping mice.

The authors first showed that they could induce false memories in awake mice. They recorded from neurons in area CA1 of the hippocampus as mice explored an arena, analyzing spiking activity online to identify putative place cells. Using a brain-computer interface (BCI), they could pair activity of a particular place cell with stimulation of the medial forebrain bundle (MFB), which is known to be rewarding, with a very short latency (less than $2 \mathrm{~ms}$ ). Such use of the BCI led mice to linger in the location encoded by the place cell paired with MFB stimulation, and their preference for this location remained even after the BCI was shut off.

In a second cohort of mice, the authors identified place cells while mice explored the arena, but they then allowed the animals to sleep. The BCI was then switched on, again pairing activity of a particular place cell with MFB stimulation, all while the mice slept. When mice were placed back in the arena after they woke up, mice went directly to the location encoded by the place cell whose activity was paired with MFB stimulation, and continued to display a preference for this location throughout the trial.

These results show that it is possible to induce new memories during sleep in mice. While previous studies in humans have shown that classical conditioning and implicit memories can be formed during sleep, this study goes a step further to show that an explicit memory leading to goal-directed behavior can be induced during sleep, and that this can be done using neural recordings and brain stimulation, bypassing sensory inputs. Further, because the memory induced by the authors here relied on activity of place cells that was uncoupled from the animal's actual position, this study provides the strongest evidence yet that place cells have a causal role in spatial memory and navigation.

Brigitta Gundersen 